\title{
Um modelo de simulação baseado em Raciocínio Qualitativo para a educação em Ciências
}

Paulo Vitor Teodoro de Souza $\frac{\text { paulovitorteodoro@yahoo.com.br }}{\text { Instituto Federal Goiano (IF Goiano), }}$ Instituto Federal Goiano (IF Goiano),
Catalão, Goiás/ Universidade de Brasilia (UnB), Brasília, Distrito Federal, Brasil

Paulo Salles

pssalles@gmail.com

Universidade de Brasília (UnB), Brasília Distrito Federal, Brasil

Ricardo Gauche

ricardogauche@gmail.com Universidade de Brasília (UnB), Brasília, Distrito Federal, Brasil

\section{RESUMO}

O presente trabalho apresenta resultados preliminares de uma pesquisa que busca investigar a efetividade de materiais didáticos fundamentados em técnicas desenvolvidas em Raciocínio Qualitativo (RQ), área da Inteligência Artificial que utiliza o raciocínio simbólico para representar funções matemáticas sem o uso de números e com relações de causalidade explicitamente modeladas. Estudos anteriores demonstram o potencial do RQ para abordar sistemas dinâmicos por meio de atividades que exploram, com o apoio de modelos de simulação, o uso de raciocínio hipotético - dedutivo para analisar conceitos complexos, e ampliam o leque de materiais e atividades disponíveis para os professores. Para tanto, construímos, na plataforma de modelagem DynaLearn (www.dynalearn.eu), um modelo qualitativo de simulação para ser usado no ensino de Ciências. Esse software é dotado de uma interface gráfica, que facilita a construção de modelos e a apresentação dos resultados de simulações. Neste trabalho descrevemos o processo de construção de um modelo qualitativo que tem o objetivo de promover a compreensão de conceitos sobre o efeito das chuvas no comportamento de uma cidade, em relação ao consumo de água potável. O modelo representa relações causais entre variáveis climáticas, vazão dos rios, o tratamento da água e o abastecimento urbano. Além de ter potencial para a utilização por estudantes ouvintes em aulas de Ciências, o modelo também pode ser empregado na educação de surdos, devido à maneira como explora os recursos visuais.

PALAVRAS-CHAVE: Material de Ensino. Raciocínio Qualitativo. Ensino de Ciências. 


\section{INTRODUÇÃO}

Entre outros fatores que dificultam o desenvolvimento de intervenções didático-pedagógico em sala de aula, frequentemente é citada a ausência de materiais de ensino que contribuam efetivamente nas ações didáticas dos professores. Reis e Silva (2012) e Nadolny (2012) apontam, inclusive, a necessidade de a comunidade científica se envolver em investigações que geram materiais didáticos para o ensino de Química, Física e Biologia. Nesse contexto, cresce a importância de pesquisas que resultam em estratégias para a educação em Ciências, e mais, que estas tenham como foco o uso de ferramentas que permitam a construção e a reconstrução de conhecimentos e conceitos a serem comunicados ou a serem criados pelos próprios estudantes.

Com efeito, o uso de modelos de simulação e a própria atividade de construir tais modelos vem sendo investigada com o objetivo de desenvolver habilidades científicas nos estudantes e de dar liberdade ao docente para construir seus próprios materiais (MIODUSER et al., 2012). O uso de modelos de simulação permite ir além do que é apresentado nos livros didáticos. É possível contextualizar, problematizar, explicar mecanismos com base no funcionamento das partes do sistema, explicar fenômenos relacionados a esses componentes, e ainda prever os possíveis resultados.

Entretanto, muitas vezes o professor se encontra limitado pelo fato de que muitos estudantes têm grandes dificuldades para visualizar os aspectos dinâmicos do funcionamento de sistemas complexos, e não dominam as bases necessárias para explorar o uso de funções matemáticas e de compreender relações quantitativas.

Nesse sentido, foi desenvolvido, por meio de colaboração estabelecida entre pesquisadores europeus e de outros países, inclusive o Brasil (ALVES, 2015), o software DynaLearn (www.dynalearn.eu). O referido software é uma plataforma de modelagem que utiliza técnicas desenvolvidas por uma área da Inteligência Artificial conhecida por Raciocínio Qualitativo (RQ), que mostra, diagramaticamente, possíveis relações causais entre os assuntos trabalhados e a previsão de resultados de simulações. O DynaLearn possibilita adquirir conhecimentos conceituais por meio da construção de modelos qualitativos e simulação de como os sistemas se comportam (BREDEWEG et al. 2013).

Diante disso, pesquisas como as descritas em Feltrini (2009), Resende (2010), Bastos (2014), Alves (2015), Cavalcante (2015), Souza, Salles e Gauche (2016), demonstram que materiais didáticos, baseado em $\mathrm{RQ}$, têm potencial para favorecer o processo de ensino e aprendizagem, e que o mesmo material pode atender tanto a estudantes surdos como ouvintes (RESENDE, 2010). Entretanto, um dos problemas que enfrentamos é a pouca quantidade de estudos voltados para o ensino de Química e para a efetiva utilização de modelos qualitativos em sala de aula.

Ferreira et al. (2014) mostraram que ainda há poucas pesquisas que favorecem o processo de inclusão de surdos, e Pereira et al. (2011) avançaram em estudos sobre interações pedagógicas mediadas pela visão no ensino de Química. Entretanto, como mostraram Souza et al. (2016), é ainda menor a quantidade trabalhos que enveredaram para a produção de materiais didáticos voltados para o ensino de Ciências na perspectiva da inclusão. Nesse sentido, trabalhos que 
utilizam modelos qualitativos de simulação para o ensino de conceitos científicos em questões ambientais a partir de aspectos visuais (FELTRINI, 2009; RESENDE, 2010; ALVES, 2015), e adicionalmente abordam o desenvolvimento do raciocínio lógico hipotético-dedutivo, podem servir de base em estudos com potencial para produzir resultados importantes para o ensino de Química.

Nesse contexto, o presente trabalho apresenta resultados preliminares de uma pesquisa cujos objetivos são: (a) investigar como o processo de elaboração e o uso de materiais didáticos para o ensino de Química baseados em RQ podem contribuir para favorecer a inclusão de estudantes surdos e ouvintes; (b) verificar a eficácia de modelos qualitativos de simulação como os materiais didáticos na superação de um ensino baseado na memorização, conteudista e descontextualizado que ainda prevalece nas escolas de educação básica brasileiras (SOUZA et al. 2015). A seguir, serão discutidos aspectos gerais do Raciocínio Qualitativo. Depois, faremos uma apresentação de DynaLearn e dos elementos básicos de um modelo qualitativo construído nessa plataforma. Em seguida, serão apresentadas uma metodologia para modelagem, e os resultados obtidos na construção do modelo 'Chuva e Água Potável', com três simulações. Finalmente, serão apresentadas a discussão dos resultados e as considerações finais.

\section{RACIOCÍONIO QUALITATIVO}

A compreensão de conceitos e fenômenos que exigem raciocínios complexos, especialmente em ciências exatas como a Química e a Física, acarretam dificuldades para muitos dos estudantes da educação básica que, talvez por atraso de aprendizagem, acumulam um déficit acadêmico durante a trajetória escolar (ALVES, 2015). Com isso, uma área da Inteligência Artificial, denominada Raciocínio Qualitativo, busca desenvolver o raciocínio simbólico para representar funções matemáticas, sem o uso de dados numéricos.

A partir de técnicas desenvolvidas em $R Q$, modelos de simulação podem ser construídos para representar a estrutura e o funcionamento de sistemas diversos, especialmente aqueles que possuem relações de causa e efeito. O RQ também é útil em situações que não exigem respostas exatas, nas quais tendências de mudança e conhecimento quantitativo expressos em linguagem cotidiana, não numérica, são suficientes. $R Q$ vem sendo empregado também no estudo de previsões, em termos genéricos, do comportamento de sistemas físicos, com base na representação da estrutura dos sistemas modelados (CAVALCANTE, 2015).

O RQ favorece a representação conceitual de sistemas, especificando suas propriedades contínuas, tais como quantidades, espaço e tempo, e usando distinções qualitativas, conceitualmente relevantes (LIEM, 2013). Uma das teorias mais importantes em RQ é a Teoria Qualitativa dos Processos (TQP), desenvolvida por Ken Forbus no início dos anos 1980. Nessa teoria, os sistemas são conjuntos constituídos por objetos, representados pelas entidades, que formam o sistema que está sendo modelado qualitativamente. Processos físicos, químicos e biológicos são identificados como as causas de mudança do sistema (LIEM, 2013; CAVALCANTE, 2015). As entidades se relacionam de tal modo que os estados de cada objeto dependem dos estados dos outros objetos. As entidades e as relações entre elas constituem a estrutura básica de um sistema, e os processos criam a dinâmica que constitui o funcionamento do sistema. Por contribuírem para 
aumentar a compreensão da estrutura e do funcionamento de sistemas dessa forma, modelos qualitativos têm sido considerados um tipo de modelos conceituais.

Assim, modelos baseados em RQ representam a dinâmica de sistemas, bem como tratam explicitamente das relações causais. Com essa base de causalidade, modelos qualitativos permitem tanto explicar o funcionamento como prever o comportamento do sistema, dadas as condições iniciais das simulações. Essas características favorecem aplicações em atividades educacionais, a partir das quais se pode estimular o desenvolvimento de raciocínio hipotético-dedutivo e da criatividade do usuário (SALLES et al., 2004; ALVES, 2015).

Além de aplicações em engenharia e outras áreas tecnológicas, o RQ tem sido tradicionalmente empregado para fins educacionais, inclusive na educação secundária e na superior (BREDEWEG, LIEM e NICOLAOU, 2016). Bredeweg et al. (2016) estão investigando como o RQ pode ser utilizado como um instrumento operacional, em tempo real, para avaliação formativa.

Crouse e Forbus (2016) também desenvolveram a pesquisa construindo modelos qualitativos, no contexto da educação primária (elementary school), nos Estados Unidos. Os autores mostram que elaborar materiais didáticos que exploram habilidades cognitivas de alta complexidade não é tarefa simples. E, nesse sentido, os autores explicitam o potencial do RQ para a utilização de modelos sobre questões cotidianas de estudantes norte-americanos. O RQ também tem contribuído na representação, por estudantes, de sistemas dinâmicos de forma qualitativa, o que também auxilia na formação do pensamento científico desses estudantes (MIODUSER et al., 2005).

No contexto brasileiro, há pesquisas que se apropriam do RQ para o ensino de Ciências. Bastos (2014) e Alves (2015) utilizaram modelos qualitativos para representação de fenômenos ecológicos. Entre materiais didáticos voltados para aspectos conceituais da Ecologia, Alves (2015) utilizou materiais pedagógicos baseados em silogismos para explorar o raciocínio dedutivo sobre conceitos expressos em modelos qualitativos. Cavalcante (2015) explorou, em sua pesquisa baseada em RQ, conteúdos de ciências, como a visão de madeireiros e de ambientalistas sobre a biodiversidade, doenças do sistema circulatório humano, aparelho reprodutor masculino, aparelho reprodutor feminino e gravidez.

Outra linha de aplicações de modelos qualitativos para fins de pesquisa científica tem produzido bons resultados. Como exemplo, Leão (2011) elaborou modelos qualitativos para representar desenvolvimento das principais teorias de metapopulações, as quais são úteis para comparar hipóteses e pressupostos envolvidos nos diferentes pontos de vista sobre esse tema. Diante disso, é possível perceber que o desenvolvimento de DynaLearn abre novas possibilidades para aplicações educacionais, algumas das quais são discutidas neste artigo.

\section{AMBIENTE DE MODELAGEM E SIMULAÇÃO: DYNALEARN}

Para a elaboração de materiais didáticos baseados em $R Q$, no presente trabalho utilizamos o ambiente de aprendizagem DynaLearn. Este software foi implementado em Prolog, uma linguagem simbólica de programação, baseada em 
lógica de primeira ordem, na qual operações de raciocínio dedutivo são efetuados. Essas operações não estão acessíveis ao usuário do software.

O DynaLearn oferece seis ambientes de modelagem, os Espaços de Aprendizagem (em inglês, Learning Spaces - LS), nos quais estão disponíveis cada vez mais elementos, com os quais se pode construir modelos e obter simulações em diferentes níveis de complexidade. No presente trabalho, usaremos apenas o Espaço de Aprendizagem denominado "Modelo causal básico, com grafo de estados" ou LS3. A descrição de todos os Espaços de Aprendizagem pode ser encontrada em Bredeweg et al. (2013).

DynaLearn apresenta uma interface gráfica que facilita a construção de modelos a partir da seleção de ícones, aos quais estão associados elementos do modelo, tais como entidades (os objetos que compõem o sistema) e configurações (vinculação entre objetos); quantidades (variáveis que representam propriedades contínuas dos objetos do sistema [as entidades]) e os respectivos espaços quantitativos (conjunto de possíveis valores qualitativos que as variáveis podem assumir); e as influências (que estabelecem as relações causais entre as quantidades, explicitam quais são as quantidades influenciadoras e as influenciadas, e o tipo de influência). Esses elementos serão detalhados a seguir.

As figuras abaixo ilustram os elementos fundamentais de um modelo qualitativo: entidades, configurações, quantidades, espaços quantitativos e influências (Figura 1). Além desses, serão apresentados também os elementos que compõem as simulações: cenário inicial (Figura 2), grafo de estados (Figura 3) e história dos valores das quantidades (Figura 4). Todas as figuras mostram imagens geradas nas telas de DynaLearn.

O modelo 'Demonstração' (Figura 1) representa um sistema com duas entidades (A-entidade e B-entidade), unidas pela configuração Vinculada a, que devem ser lidas como essas duas entidades estão relacionadas: "A-entidade é vinculada a B-entidade". A cada entidade, está associada a uma quantidade, que representa no modelo uma propriedade contínua (por exemplo, massa, altura, volume, etc.). Os valores das quantidades podem ter dois componentes: magnitude (o 'tamanho' do objeto) e a derivada (a 'direção' da variação, se existir).

Nesse modelo, a entidade 'A-entidade' está associada à Quantidade-a. O espaço quantitativo associado a ela é constituído apenas da derivada, que pode ter os valores incluídos no conjunto \{crescendo, estável (zero), diminuindo\}. Todas as derivadas representadas em DynaLearn possuem esse espaço quantitativo. A 'B-entidade', por sua vez, está associada à Quantidade- $b$. Os espaços quantitativos associados a esta quantidade são constituídos por dois componentes, magnitude e derivada. A magnitude de Quantidade- $b$ pode assumir os valores do espaço quantitativo \{inferior, limite, superior\}. A figura mostra que este espaço quantitativo é constituído de intervalo (inferior), ponto (limite) e intervalo (superior). Todos os espaços quantitativos de DynaLearn apresentam valores contínuos, com alternância de pontos e intervalos. 
Figura 1. Modelo 'Demonstração'

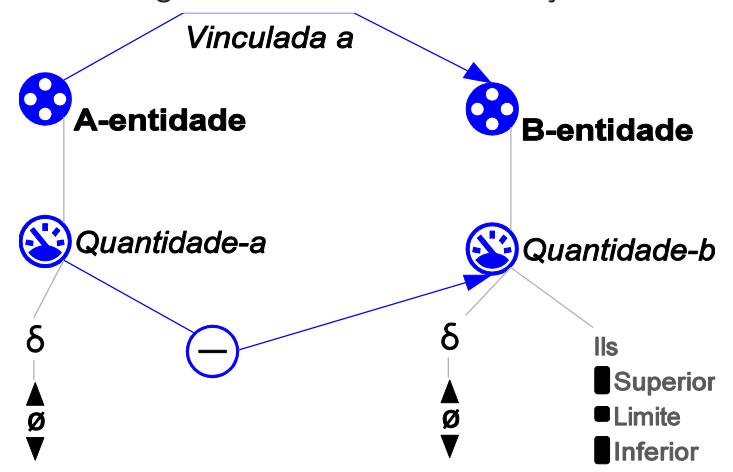

Fonte: Imagem gerada pelo software DynaLearn

Além desses elementos, o modelo possui uma influência entre Quantidade-a e Quantidade- $b$. O sentido da seta mostra que a primeira é 'influenciadora' e a segunda, 'influenciada'. Nessa figura, o tipo de influência é 'negativa', o que significa: quando a quantidade influenciadora estiver variando, a quantidade influenciada será compelida a variar na direção oposta. Por exemplo, se Quantidade- $a$ estiver crescendo, Quantidade- $b$ vai diminuir. Alternativamente, influências 'positivas' indicam que as quantidades, influenciadora e influenciada, variam na mesma direção.

A Figura 2 mostra o primeiro passo para a simulação: a construção de um cenário, no qual são indicados os 'valores iniciais' das quantidades. No caso, Quantidade- $a$ está crescendo, e Quantidade- $b$ possui magnitude com valor qualitativo no intervalo 'superior', e derivada indefinida. Sendo a influência entre elas negativa, a derivada de Quantidade-b assumirá valor oposto ao de Quantidade- $a$ e a quantidade influenciada vai diminuir. Nessa situação, em algum momento o valor de Quantidade-b chegará ao ponto 'limite' e, caso a simulação continue, passará para o intervalo 'inferior' ao ponto 'limite'.

Figura 2. Cenário do modelo 'Demonstração'

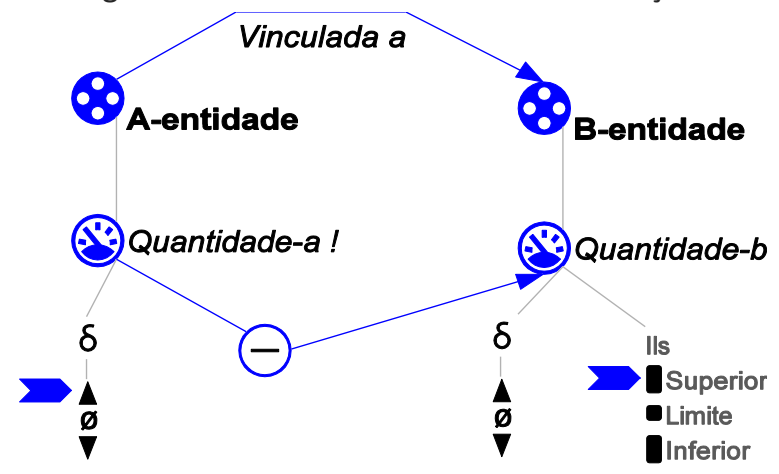

Fonte: Imagem gerada pelo software DynaLearn

Do ponto de vista qualitativo, o sistema passa pelos seguintes estados: aquele do cenário, em que o valor de Quantidade- $a$ está definido, e o da Quantidade-b está parcialmente definido, pois apenas a magnitude desta quantidade possui um valor atribuído. O primeiro estado definido durante a simulação é aquele em que a Quantidade- $b$ terá definida sua derivada. Deste estado, é possível gerar outro em que o valor da magnitude de Quantidade- $b$ muda e chega ao valor 'limite' e, em seguida, a outro estado, no qual a magnitude desta quantidade chega ao intervalo 
'inferior'. O grafo de estados desta simulação, portanto, terá visualmente quatro elementos: um cenário, e três estados identificados por números, como mostra a Figura 3.

A Figura 4, por sua vez, mostra a história dos valores assumidos pelas duas quantidades nos estados produzidos pela simulação. 0 diagrama dos valores de cada quantidade mostra o valor da magnitude e o da derivada. Os resultados da simulação são, portanto, exibidos nesses diagramas e podem ser lidos como segue: Quantidade- $a$ assume, nos estados 1, 2 e 3 o mesmo valor, (crescendo). A Quantidade-b apresenta no estado 1, os valores (superior, diminuindo); no estado 2. (limite, diminuindo); e no estado 3, (inferior, diminuindo).

Figura 3. Grafo de estados resultante de simulação com o modelo 'Demonstração'

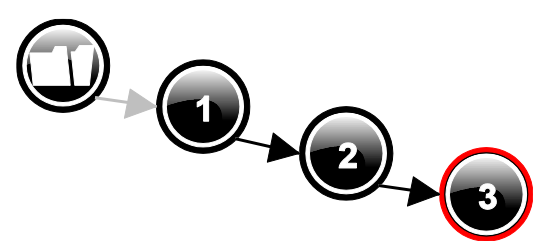

Figura 4. História dos valores das quantidades em simulação com o modelo 'Demonstração'

\section{A-entidade: Quantidade-a}

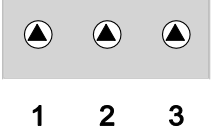

B-entidade: Quantidade-b

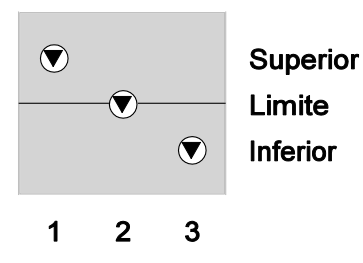

Fonte: Imagem gerada pelo software DynaLearn

Concluindo, o modelo representa um sistema e seu comportamento (as mudanças descritas na sequência de estados). A passagem do tempo, embora não medida quantitativamente, indica que o estado 1 é desdobramento do cenário, e é seguido pelos estados 2 e 3 , indicando fenômenos que ocorrem em intervalos de tempo diferentes (não quantificados). Admite-se que a duração de um estado em que o valor de uma quantidade está em um intervalo seja maior que a duração de outro, em que o valor está em um ponto. Sair de um intervalo deve demorar mais que deixar um ponto.

\section{METODOLOGIA PARA A CONSTRUÇÃO DO MODELO}

Depois de estudar o sistema a ser modelado, e identificar relações de causa e consequência capazes de mudar as condições iniciais, elaboramos um pequeno texto motivador denominado "Chuva e Água Potável", que descreve o efeito da chuva sobre o comportamento da população em relação ao consumo de água. A criação desse texto motivador tem o objetivo de nortear conceitualmente a elaboração do modelo e a simulação em DynaLearn.

O modelo, criado para ser material didático, foi elaborado no LS3. Optamos por construir o modelo qualitativo nesse espaço de aprendizagem para possibilitar a inclusão de espaços quantitativos, com derivada e magnitude, no sistema 
elaborado. Essas informações ampliam as possibilidades de trabalhar os conhecimentos propostos. No espaço de aprendizagem L2, quantidades têm apenas derivadas e, nos níveis LS4, LS5 e LS6, as quantidades possuem magnitudes e derivadas. Entretanto, esses modelos, mais complexos, são mais difíceis de serem inseridos em sala de aula antes que os estudantes e/ou professores estejam familiarizados com o uso de modelos e simulações como materiais didáticos.

Em seguida, buscamos identificar, no texto, os elementos fundamentais da modelagem qualitativa (entidades, quantidades, configurações, relações de influência e espaços quantitativos) e fizemos um teste de conceitos, utilizando o software DynaLearn. Posteriormente, elaboramos no ambiente de modelagem possíveis relações causais que podem ser trabalhadas a partir do texto motivador, explorando tópicos de Ciências e Química.

Buscamos relacionar, no material didático, aspectos importantes do ensino de Ciências sobre o ciclo da água, as propriedades físico-químicas utilizadas no tratamento da água, assim como impactos no contexto social (por exemplo, o racionamento), vivenciado em várias cidades do mundo. Por fim, realizamos simulações para checar as previsões geradas pelo modelo em cenários diferentes, analisando os resultados gerados pelo modelo como material didático-pedagógico.

\section{RESULTADOS}

O material didático aqui apresentado tem por objetivo dar suporte para discussões sobre os fatores que influenciam consumo e mesmo o racionamento de água nas cidades brasileiras. $O$ intuito foi apontar possíveis relações causais sobre as variáveis que o meio ambiente (a atmosfera) pode causar na forma do consumo de água pela população. Dentre as variáveis que possuem relações causais, destacamos: vapor de água na atmosfera, chuva, volume dos rios, os processos realizados na Estação de Tratamento de Água (ETA) e o consumo de água pela população. Para a construção do modelo qualitativo, inicialmente elaboramos o texto motivador, apresentado no Quadro 1:

Quadro 1 - Texto motivador: Chuva e Água Potável

São muitos os fatores que influenciam o consumo de água nas cidades brasileiras, a São muitos os fatores que influenciam o consumo de agua nas cidades brasileiras, a
começar por aspectos climáticos e ambientais. A atmosfera é constituída por diversos componentes e, entre eles, a água no estado vapor. Quando as nuvens estão carregadas de água e as condições são adequadas para a condensação, ocorre a precipitação na forma de chuva. Assim, dependendo da quantidade de vapor de água na atmosfera e de chuva, o volume dos rios das cidades, que abastecem a área urbana, será alterado. Logo, a vazão da água captada pela Estação de Tratamento de Água (ETA) também pode ser modificada. Desse modo, a distribuição de água pela ETA para a área urbana também depende da quantidade de vapor de água na atmosfera. A água potável distribuída precisa, segundo a Organização Mundial de Saúde (OMS), seguir alguns padrões apontados por testes físicoquímicos de qualidade, tais como odor, turbidez e pH. É importantíssimo que a ETA respeite esses padrões, uma vez que o consumo humano de água não potável pode causar malefícios para a saúde da população. Por exemplo, se a turbidez não estiver controlada e a água estiver opaca, isso indica a possível presença de impurezas que podem causar ou transmitir doenças. Para isso, utilizam-se produtos químicos no tratamento, que atuam no sentido de reduzir o odor e a turbidez, tal que quanto maior a quantidade de produtos químicos utilizados, menor será o odor e a turbidez (certamente existem limites para os 
produtos químicos utilizados, de acordo com o volume de água a ser tratado). Entretanto, a necessidade de elevar o $\mathrm{pH}$ (reduzindo a acidez) requer maior quantidade de produtos químicos, de maneira que os valores das duas quantidades variam no mesmo sentido. Essas propriedades da qualidade da água tratada influenciam, portanto, a quantidade de água potável. E esta, por sua vez, influencia no mesmo sentido, o consumo de água pela população, que poderá ser de forma racionada, limitada, consciente e até livre.

Fonte: Autoria própria (2017).

A partir da elaboração do texto motivador, iniciamos o planejamento da modelagem no software DynaLearn. Para isso, identificamos as seguintes entidades no texto motivador: Atmosfera, Rio, Área urbana, ETA, Água produzida, População. Posteriormente, apontamos as possíveis configurações entre as entidades vinculadas. Esses elementos são apresentados no Quadro 2:

Quadro 2: Entidades e configurações

\begin{tabular}{l|l|l}
\hline $\begin{array}{l}\text { Entidade } \\
\text { influenciadora }\end{array}$ & Configuração & Entidade influenciada \\
\hline Atmosfera & Altera o volume & Rio \\
\hline Rio & Abastece & Área urbana \\
\hline Área urbana & É ocupada & População \\
\hline Área urbana & Trata água & ETA \\
\hline ETA & Distribui & Água produzida \\
\hline
\end{tabular}

Fonte: Autoria própria (2017).

Em seguida, foram identificadas as quantidades associadas às entidades que compõem a estrutura do sistema, aquelas que representam características variáveis das entidades: Vapor de água, Chuva, Vazão captada pela ETA, Água potável, Produtos químicos, Turbidez, pH, Odor e Consumo. As entidades e respectivas quantidades relacionadas encontram-se no Quadro 3:

Quadro 3: Entidades e respectivas quantidades

\begin{tabular}{l|l}
\hline \multicolumn{1}{c|}{ Entidade } & Quantidade \\
\hline Atmosfera & $\begin{array}{l}\text { Vapor de água } \\
\text { Chuva }\end{array}$ \\
\hline Rio & Vazão captada pela ETA \\
\hline Área urbana & Água potável \\
\hline ETA & Produtos químicos \\
\hline Água Produzida & $\begin{array}{l}\text { Turbidez } \\
\text { pH } \\
\text { Odor }\end{array}$ \\
\hline
\end{tabular}

Fonte: Autoria própria (2017).

É necessário caracterizar bem o significado de cada variável, para maior clareza dos conhecimentos representados no modelo e nos resultados produzidos pelas simulações. No Quadro 4, apontamos as entidades, quantidades e o respectivo significado de cada quantidade: 
Quadro 4: Descrição das quantidades associadas às entidades

\begin{tabular}{|c|c|c|}
\hline Entidade & $\begin{array}{l}\text { Quantidad } \\
\text { e }\end{array}$ & Explicação da quantidade \\
\hline Atmosfera & Vapor de água & $\begin{array}{l}\text { Retrata a quantidade de umidade presente na atmosfera. } \\
\text { O vapor de água é levado para as altitudes, pelo vento } \\
\text { vertical, e, por resfriamento, ocorre a condensação, } \\
\text { seguida da precipitação na forma de chuva, que contribui } \\
\text { para o aumento da água nos rios. }\end{array}$ \\
\hline Atmosfera & Chuva & $\begin{array}{l}\text { Representa a quantidade de água líquida formada a } \\
\text { partir do vapor de água presente na atmosfera; a água da } \\
\text { chuva tem potencial para se infiltrar no solo, se deslocar } \\
\text { por escoamento superficial e afetar a vazão de um rio. }\end{array}$ \\
\hline Rio & $\begin{array}{l}\text { Vazão captada } \\
\text { pela ETA }\end{array}$ & $\begin{array}{l}\text { Descreve o volume de água que a Estação de Tratamento } \\
\text { recebe do(s) rio(s) da região. Esse processo de captação } \\
\text { resulta em aproveitamento da água que chega aos rios } \\
\text { por meio das chuvas ou de nascentes (que também } \\
\text { podem resultar de água das chuvas). Essa vazão de água } \\
\text { afetará a quantidade de água que a população pode } \\
\text { consumir. }\end{array}$ \\
\hline ETA & $\begin{array}{l}\text { Produtos } \\
\text { químicos }\end{array}$ & $\begin{array}{l}\text { Corresponde à quantidade do conjunto de diversas } \\
\text { substâncias que a ETA adiciona à água para realizar o } \\
\text { tratamento e, assim, garantir a potabilidade para o } \\
\text { consumo da população. }\end{array}$ \\
\hline $\begin{array}{l}\text { Água } \\
\text { produzida }\end{array}$ & Turbidez & $\begin{array}{l}\text { Representa a quantidade de partículas em suspensão na } \\
\text { água, que podem reduzir a limpidez da água. A presença } \\
\text { de impurezas, como terra, detritos, folhas, entre outras, } \\
\text { torna a água mais opaca e, consequentemente, mais } \\
\text { túrbidas, em relação à água sem impurezas. Portanto, } \\
\text { essa é uma variável que condiciona a qualidade da água } \\
\text { para ser consumida. }\end{array}$ \\
\hline $\begin{array}{l}\text { Água } \\
\text { produzida }\end{array}$ & $\mathrm{pH}$ & $\begin{array}{l}\text { A quantidade "pH" representa o índice de acidez, ou } \\
\text { basicidade, da água. Quanto menor o valor numérico do } \\
\mathrm{pH} \text {, maior será a acidez. Em contraposição, quanto maior } \\
\text { o valor numérico do } \mathrm{pH} \text {, menor será a acidez da água, ou } \\
\text { maior será a alcalinidade. O valor do pH é controlado por } \\
\text { meio de produtos químicos e depende do volume de } \\
\text { água a ser tratada. }\end{array}$ \\
\hline $\begin{array}{l}\text { Água } \\
\text { produzida }\end{array}$ & Odor & $\begin{array}{l}\text { Também equivale a uma quantidade variável da água. } \\
\text { Quando esta chega a ETA, por meio da captação dos rios, } \\
\text { pode apresentar odor característico de substâncias em } \\
\text { decomposição. Com o uso de produtos químicos, o odor } \\
\text { poderá ser controlado e mesmo se tornar inodora, para } \\
\text { garantir a potabilidade e a utilização da água pela } \\
\text { população. }\end{array}$ \\
\hline $\begin{array}{l}\text { Área } \\
\text { urbana }\end{array}$ & Água potável & $\begin{array}{l}\text { Caracteriza, no modelo, a quantidade de água própria } \\
\text { para o consumo da população, que chega à área urbana. }\end{array}$ \\
\hline População & Consumo & $\begin{array}{l}\text { Refere-se à disponibilidade de água que a população } \\
\text { pode consumir e, dependendo da umidade do ar, das } \\
\text { chuvas, da vazão captada nos rios, dos produtos químicos } \\
\text { utilizados e das propriedades da água produzida (como } \\
\text { turbidez, pH, odor). A quantidade efetivamente } \\
\text { consumida pela população varia desde um valor definido } \\
\text { (racionamento) até o consumo livre. }\end{array}$ \\
\hline
\end{tabular}


Com as entidades e quantidades identificadas, é possível iniciar a modelagem em DynaLearn. Inserimos as entidades com as respectivas configurações e, em seguida, as quantidades de cada entidade. Posteriormente, são estabelecidas as influências. A Figura 5 mostra o modelo 'Chuva e Água Potável', que pode ser utilizado como material didático. (Observação: devido às características da implantação do software, todas os nomes das variáveis de modelos construídos em DynaLearn devem começar com letras maiúsculas. Por esse motivo, ao invés de $\mathrm{pH}$, a variável é identificada nas figuras como $\mathrm{Ph}$ ).

Figura 5: Modelo elaborado: Chuva e Água Potável

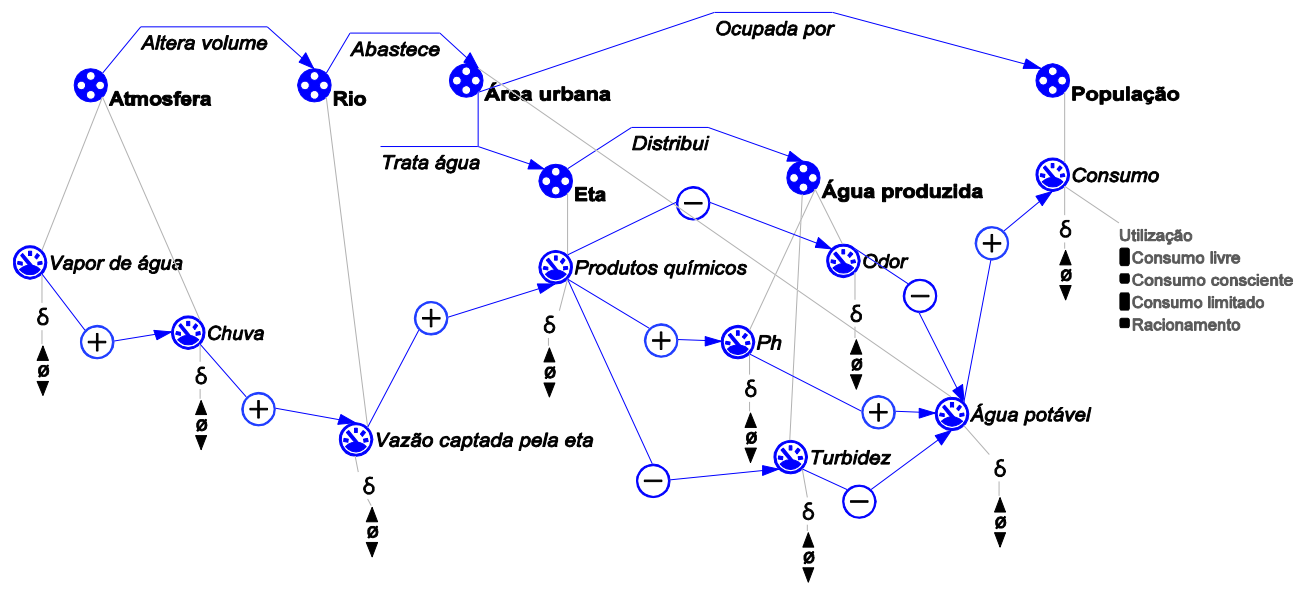

Fonte: Imagem gerada pelo software DynaLearn

Na Figura 5, podemos perceber, de forma mais clara, as relações causais entre as quantidades que expressam a influência das chuvas sobre o consumo de água pela população. É possível notar que o vapor de água influencia na quantidade de chuva, tal que as duas mudam na mesma direção (seta com sinal +). A vazão de água captada pela ETA e os produtos químicos necessários para o tratamento são influenciados, na mesma direção, pela quantidade de chuva. A influência que os produtos químicos causam sobre a quantidade odor e turbidez faz com que essas mudem na direção inversa (seta com sinal -) à de sua influenciadora, ao contrário do que acontece com o $\mathrm{pH}$, que segue na mesma direção dos produtos químicos. Turbidez e odor influenciam, em sentidos opostos, a quantidade de água potável. Por sua vez, a quantidade de água potável varia na mesma direção do consumo de água pela população. Em resumo, dependendo do valor inicial da derivada da quantidade Vapor de água, o consumo de água da população poderá ser alterado.

Tendo sido o modelo gerado no LS3, é possível que as variáveis tenham apenas as derivadas. Entretanto, no modelo 'Chuva e água potável' definimos a magnitude da variável Consumo, associada ao seguinte espaço quantitativo: \{racionamento, consumo limitado, consumo consciente e consumo livre\}.

Conforme mencionado acima, o modelo elaborado pode ter vários cenários iniciais para simulações, dependendo dos valores iniciais das variáveis Vapor de água e Consumo. Para fins de ilustração, o modelo pode ser usado com três (3) cenários, dependendo dos valores iniciais dessas quantidades: a) Vapor de água= (crescendo) e Consumo $=($ racionamento $) ;$ b) Vapor de água $=($ diminuindo $)$ e Consumo $=($ consumo livre $) ; \mathrm{c})$ Vapor de água $=($ estável $)$ e Consumo $=($ consumo livre). Esses cenários serão comentados a seguir. 


\section{SIMULAÇÃO COM A QUANTIDADE DE VAPOR DE ÁGUA ATMOSFÉRICO AUMENTANDO}

Nesta primeira simulação, atribui-se à derivada de Vapor de água o valor inicial crescendo. O valor inicial da magnitude de Consumo é racionamento, e sua derivada não é definida. Na Figura 6, este cenário é apresentado. Nota-se que o modelo qualitativo traz os respectivos valores iniciais da simulação representados por uma seta azul e pelo sinal de exclamação (!) nas variáveis às quais foram atribuídos valores iniciais.

Figura 6: Modelo 'Chuva e Água Potável': cenário com valor inicial crescendo na derivada de Vapor de água, e valor inicial racionamento na magnitude de Consumo.

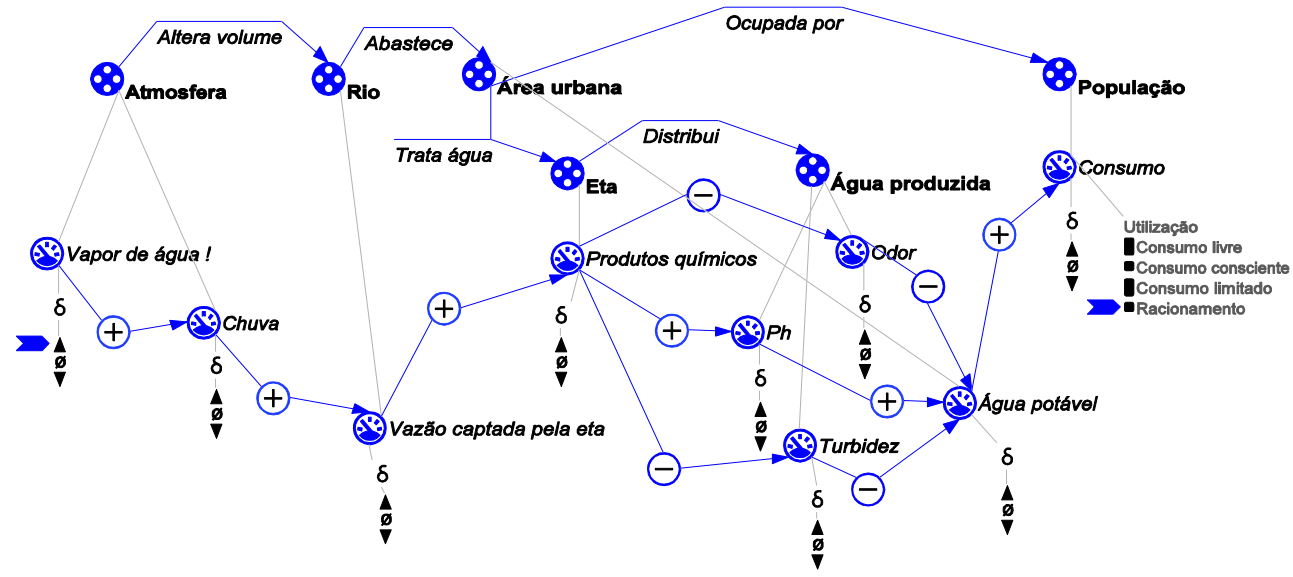

Fonte: Imagem gerada pelo software DynaLearn

A Figura 7 mostra o grafo de estados, diagrama que representa o cenário descrito e os quatro estados produzidos na simulação. Esse é um dos possíveis comportamentos do sistema, que depende dos valores iniciais atribuídos às variáveis Vapor de água e Consumo, e as transições entre os quatro estados.

Figura 7: Representação do grafo de estados com a derivada inicial crescendo

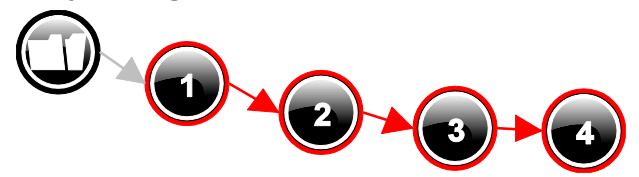

Fonte: Imagem gerada pelo software DynaLearn

Os valores assumidos por todas as quantidades representadas no modelo durante a simulação são apresentados na Figura 8. Esses diagramas da história dos valores das quantidades permitem compreender as mudanças ocorridas nas transições entre os estados: [estado $1 \rightarrow$ estado $2 \rightarrow$ estado $3 \rightarrow$ estado 4]. 
Figura 8: Resultados da simulação considerando a derivada inicial crescendo

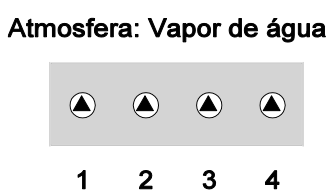

Atmosfera: Chuva

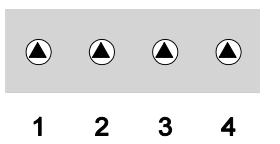

Rio: Vazão captada pela eta

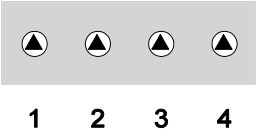

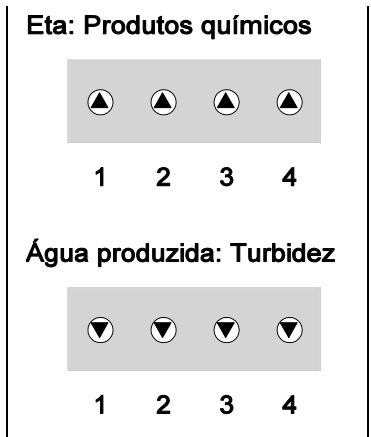

Água produzida: $\mathrm{Ph}$

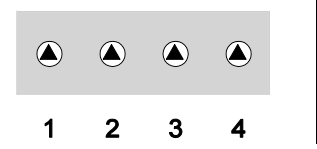

Água produzida: Odor

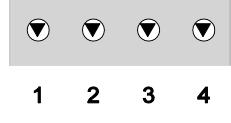

Área urbana: Água potável

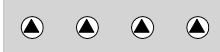

$\begin{array}{llll}1 & 2 & 3 & 4\end{array}$

População: Consumo

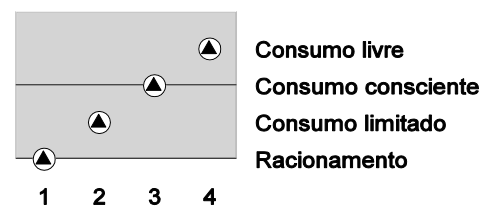

Fonte: Imagem gerada pelo software DynaLearn

No cenário desta simulação, consideramos que a quantidade de vapor d'água na atmosfera estava crescendo. No estado 1 da simulação, nota-se que as influências entre as quantidades propagaram o valor dessa derivada para todas as quantidades do sistema conforme o tipo de influência (positiva ou negativa). Esse efeito torna-se mais visível na quantidade Consumo. Embora o valor da magnitude continue em racionamento, o triângulo dentro do círculo indica que esta variável também ganhou a derivada com valor crescendo.

Como todas as demais quantidades têm apenas a representação da derivada, no estado 2 estas continuam na mesma situação em que se encontravam no estado 1 . Seus valores continuam crescendo ou decrescendo durante o estado 2 , ainda que suas magnitudes não estejam sendo consideradas. Neste estado, muda apenas a magnitude de Consumo, que passa para o intervalo consumo limitado, e aí permanece durante um certo intervalo de tempo.

A certa altura da simulação, enquanto as demais quantidades permanecem inalteradas, a magnitude de Consumo atinge um ponto que define o que foi identificado como uma mudança significativa de comportamento, chamado de 'consumo consciente'. Esta é a principal mudança que caracteriza o estado 3. Teoricamente, imediatamente depois começa o estado 4, no qual a magnitude de Consumo adentra o intervalo de valores identificado como consumo livre. Neste, o cidadão consome água potável de acordo com suas necessidades, dentro de limites que não estão representados no modelo. No intervalo de tempo de duração do estado 4, o vapor de água na atmosfera continua crescendo, a chuva mantém esse mesmo comportamento, assim como a vazão captada do 'Rio'. A atividade da 'ETA' requer que o uso de produtos químicos permaneça crescendo, e os efeitos dessa variação são transferidos para a turbidez, o pH e o odor, de acordo com o tipo de influência (positiva ou negativa), mais uma vez, sem que limites ao comportamento dessas variáveis sejam definidos no âmbito do modelo. E assim termina a simulação, sem que uma situação de equilíbrio seja estabelecida. 


\section{RESULTADO DA SIMULAÇÃO COM O VAPOR DE ÁGUA DIMINUINDO}

Esta simulação começa com um cenário em que o modelo 'Chuva e água potável' apresenta os seguintes valores iniciais: valor da derivada de Vapor de água = diminuindo; e valor da magnitude de Consumo = consumo livre, e derivada indefinida. $O$ grafo de estados produzido nesta simulação é o mesmo mostrado na Figura 7.

Na Figura 9, segue o resultado da simulação, considerando a derivada inicial diminuindo e o espaço quantitativo, representado pelo consumo, está com valor inicial no consumo livre:

Figura 9: Resultados da simulação considerando a derivada inicial diminuindo

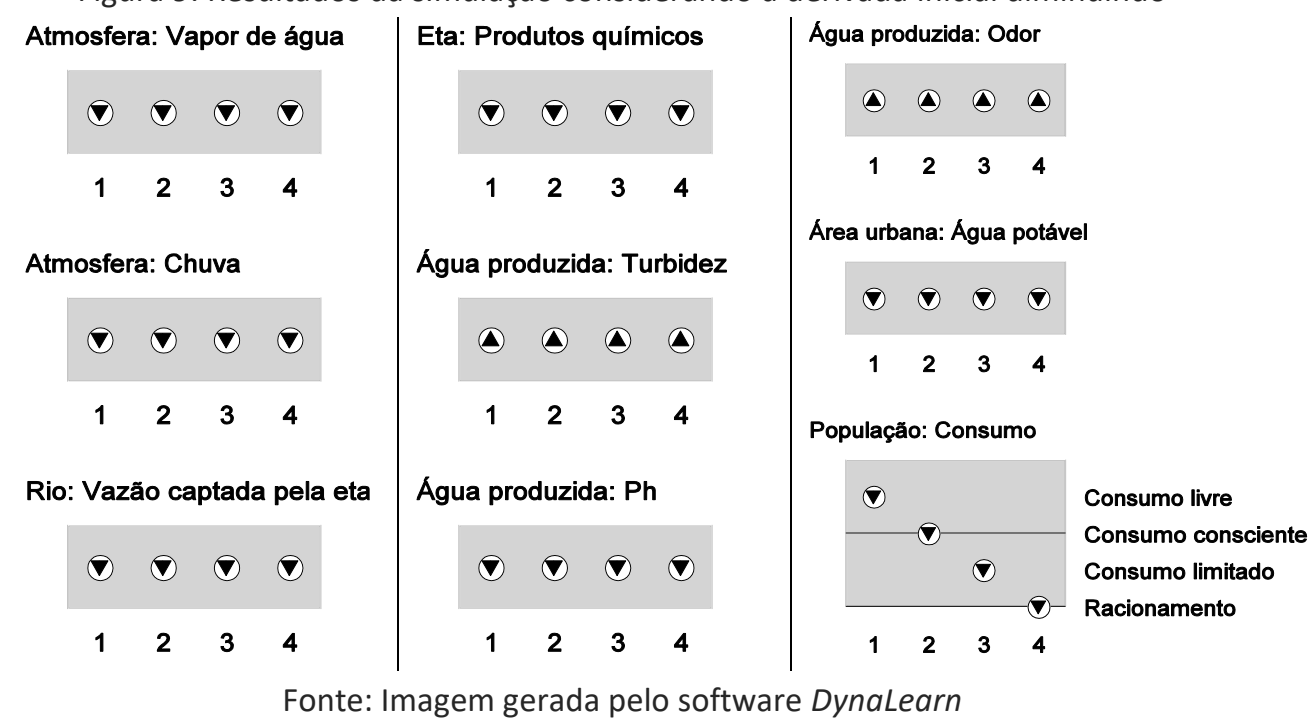

A partir do cenário mostrado na Figura 5, com derivada inicial diminuindo, podemos também entender o comportamento do sistema em quatro estados distintos. No primeiro estado, tem-se o vapor de água da atmosfera diminuindo, assim como a chuva. A vazão de água captada pela ETA também diminui e, consequentemente, a quantidade de produtos químicos, utilizados pela ETA irá diminuir. Com isso, o odor e a turbidez da água tendem a aumentar, ao contrário do $\mathrm{pH}$. Aumentando-se o odor e a turbidez, e diminuindo o $\mathrm{pH}$, pode-se diminuir a quantidade de água potável, e logo o consumo de água pela população, que no primeiro estado (o cenário inicial) é livre, com o passar do tempo diminuirá.

No estado 2, as quantidades vapor de água na atmosfera, chuva, vazão, produtos químicos, turbidez, $\mathrm{pH}$, odor e água potável mantêm as tendências observadas no estado 1 . No entanto, o consumo deixará de ser livre e chega ao ponto limite do consumo consciente. Depois desse estado, as recomendações são para que utilizem água apenas para fins imprescindíveis, como alimentação.

No estado 3 , todas as quantidades mantêm os mesmos valores, exceto o Consumo, que será limitado durante um período de tempo. Este intervalo pode representar um valor de consumo em que a coletividade passa a consumir de maneira limitada, no qual a disponibilidade de água para a população é limitada, podendo ser até racionada, caso o limite seja ultrapassado.

Finalmente, no estado 4, todas as quantidades mantêm os mesmos valores, com exceção do Consumo, que chega a um ponto em que a água passará a ser 
racionada. Com o racionamento, a água não chega às casas das pessoas em determinados dias ou períodos do dia. Neste ponto, a simulação termina.

\section{RESULTADO DA SIMULAÇÃO COM O VAPOR DE ÁGUA ESTÁVEL}

Como terceira possibilidade para o cenário, tem-se a derivada inicial estável (isto é, igual a zero). Nessa situação, a derivada de todas as variáveis assume o mesmo valor. Com a simulação representando a quantidade de vapor d'água estável, isto é, derivada zero, a simulação apresenta apenas um estado. Isso significa que, na simulação, o comportamento do sistema terá apenas um estado, e o sistema permanecerá estável até que algo aconteça, durante um intervalo de tempo. Na Figura 10 segue o grafo representando apenas um estado:

Figura 10: Representação do grafo de estados com a derivada inicial estável

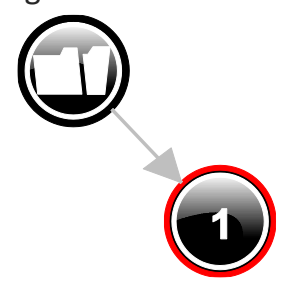

Fonte: Imagem gerada pelo software DynaLearn

Nesse caso, se o vapor de água permanece estável, a quantidade de chuva, a vazão de água captada pela ETA, a quantidade de produtos químicos, assim como o pH, odor e turbidez, também não irão sofrer modificações. Logo, a quantidade de água potável não será alterada, e o consumo permanecerá estável, com a magnitude no valor em que começou a simulação (no exemplo, consumo livre).

\section{DISCUSSÃO}

Importante ponto a ser considerado para avaliar a eficácia de materiais didáticos é a possibilidade de envolver professores e estudantes no processo de ensino e aprendizagem. Com efeito, o processo de modelagem é extremamente rico, pois requer uma grande compreensão da estrutura e do funcionamento do sistema a ser representado. Experiências realizadas por Cavalcante (2015), com estudantes do Ensino Fundamental, demonstram o potencial que tem DynaLearn como plataforma para o desenvolvimento de habilidades necessárias para a modelagem. Restou demonstrada que a relativa simplicidade no processo de construção e uso dessa plataforma para a modelagem favorece a utilização do DynaLearn como ambiente para a construção de materiais didáticos. Passado o período de adaptação ao uso do software, estudantes poderão ser capazes de expressar suas próprias ideias em modelos qualitativos, como mostraram Mioduser et al. (2012) e Cavalcante (2015).

Podemos perceber que o modelo construído no presente trabalho permitiu gerar resultados que permeiam e tomam como referencial as diferentes situações de consumo de água pela população, desde o racionamento até o consumo livre, consciente. Considerando-se as entidades, as configurações, as propriedades e os espaços quantitativos criados - característicos do LS3, é possível construir uma narrativa muito próxima do cotidiano dos discentes. Ressaltamos, inclusive, que o 
nível de complexidade LS3, comparado ao LS2, possui mais recursos (inclusive outros que não foram utilizados no presente trabalho). Entretanto, o nível LS2 também possibilita a estruturação de um modelo de causas e efeitos, por meio do diagrama de influências envolvendo apenas derivadas, mas não existe a possibilidade de gerar diferentes estados na simulação, o grafo de estados, devido à falta de representação de magnitudes nos valores das quantidades. Mas isso não quer dizer que o nível LS2 não possa dar suporte para materiais didáticos relevantes. Os trabalhos de Alves (2015) e Cavalcante (2015) utilizaram o LS2, alcançaram resultados positivos na comunicação de conceitos e ao explorar o raciocínio dedutivo dos estudantes utilizando sentenças do tipo "SE... ENTÃO..." e "Y aconteceu PORQUE X..." inspiradas no modelo e mostraram o potencial do LS2.

Nas seções anteriores, mostramos três simulações. Em uma delas, o sistema começa em equilíbrio e não se altera. Nas outras duas simulações, ao final, o sistema não atingiu o equilíbrio: no estado final, as variáveis estavam crescendo ou diminuindo. Esse final é determinado por limitações do ambiente de aprendizagem utilizado, o LS3. Para que o equilíbrio de um sistema dinâmico possa ser representado, são necessários pelo menos dois requisitos: (a) o primeiro é ter a possibilidade de representar no modelo dois fatores de causalidade distintos: (i) um elemento de modelagem que modele processos, reconhecidos como mecanismos que levam ao início de alguma mudança no sistema, ou interrompam mudanças em andamento; (ii) outro elemento que propague os efeitos dos processos para outras variáveis do sistema, a exemplo das influências positivas e negativas utilizadas no LS3 e aplicadas no modelo descrito no presente trabalho; e (b) O segundo requisito é poder representar mecanismos de retroalimentação (feedback), nos quais um fator de mudança (processo) produz efeitos que se propagam pelo sistema até chegar a uma quantidade que influencia a causa inicial da mudança. Fica claro que para modelar retroalimentação é pré-requisito ter os dois tipos de causalidade mencionados em (a). Essa modelagem é possível nos níveis LS4, LS5 e LS6.

Além de avaliar o material didático já preparado sobre o modelo 'Chuva e água potável' com estudantes e professores, a continuidade do trabalho aqui apresentado inclui a reconstrução deste modelo e a construção de outros, acompanhados dos respectivos materiais didáticos, nos níveis LS4, LS5 e LS6. Essa é outra característica enriquecedora do ambiente da pesquisa utilizado DynaLearn: possibilitar a remodelagem de materiais didáticos já elaborados, uma vez que o modelador pode encontrar possibilidades de incrementar um material didático a partir de possíveis relações de causa e efeito identificadas.

\section{CONSIDERAÇÕES FINAIS}

O modelo elaborado demonstra relações de causa e efeito em um sistema de abastecimento urbano para o consumo de água. Trabalho em andamento vai testar se esse modelo pode suscitar, em sala de aula, discussões acerca de temas específicos de Ciências (ciclo hidrológico, tratamento da água, propriedades físicoquímicas) e, ao mesmo tempo, desenvolver discussões sobre o contexto da realidade dos estudantes. As questões relacionadas à disponibilidade de água, em muitos aspectos, exigem conceitos complexos que ultrapassam os limites das Ciências Naturais e adentram as Ciências Sociais e Humanas, entre outras. 
O ciclo hidrológico é parte dos currículos da educação básica, e oferece várias oportunidades para que seja ressaltada a importância do consumo consciente. Entretanto, dificilmente são aprofundadas as relações entre fatores naturais, sociais e econômicos que condicionam o consumo de água pela população. Os modelos qualitativos contribuem para integrar o contexto social, em relação ao uso consciente da água, e contextos que envolvem conhecimentos específicos de Ciências. Esse é um dos grandes potenciais da modelagem qualitativa: a articulação em um fenômeno cotidiano e macroscópico, presente no dia a dia da população, com a justificação científica que fundamenta o fenômeno.

Apesar de os modelos contribuírem para tornar explícita a visão que se tem do sistema, faz-se necessário utilizar materiais didáticos explicativos, destinados a apresentar aos estudantes o significado de $\mathrm{pH}$, turbidez e odor e, dessa forma, desagregar os componentes que, no modelo, são representados pela quantidade Produtos químicos. Esses conceitos podem então ser contextualizados nas análises químicas utilizadas pelas ETAs para garantir a qualidade da água.

Ressalte-se ainda que os modelos qualitativos permitem a visão da dinâmica do sistema, adicionando as mudanças ao longo do tempo que afetam fenômenos complexos, discussões que dificilmente acontecem em aulas expositivas e que geram explicações desconectadas na vida real, fora da escola.

Merece destaque também o fato de que materiais didáticos baseados em modelos qualitativos podem ser destinados a atividades com estudantes do 9으 ano do Ensino Fundamental, na disciplina Ciências. Naturalmente, a complexidade dos modelos adotados deve ser dosada adequadamente, e os materiais didáticos correlatos devem ser motivadores, de modo a despertar a criatividade dos estudantes para propor alterações nos modelos apresentados, e explorar simulações alternativas. Nesse sentido, DynaLearn é uma ferramenta flexível, que permite representar o mesmo sistema em espaços de aprendizagem diferentes, e alterar o modelo com relativa simplicidade, passado o período de aprendizagem no uso dos elementos de modelagem e na manipulação da interface gráfica.

O modelo apresentado neste trabalho também poderia ser desenvolvido no Ensino Médio, especialmente nas aulas de Química. Nessa disciplina, os estudantes aprendem técnicas de análises químicas, como pH, turbidez e odor. Talvez, dependendo da instituição, não seja possível que os estudantes manipulem todas essas análises, mas, ainda assim, o professor pode relacionar as análises com o tratamento de água, com o fenômeno do ciclo hidrológico (aprendido em séries anteriores) e com o contexto social de sensibilização sobre o uso da água.

Diante disso, outro ponto enriquecedor da modelagem qualitativa no ensino de Ciências, especialmente com o modelo gerado neste trabalho, se refere na superação da compartimentalização e fragmentação dos conteúdos. A ciência não deveria ser trabalhada de forma compartimentada, sob o risco de torná-la difícil, descontextualizada, a-problemática e abstrata, o que promove visões distorcidas do que, de fato, é a ciência (CACHAPUZ et al. 2011). A modelagem qualitativa promove essa interação entre a articulação de diversos temas envolvidos em um fenômeno elaborado no material didático.

Por último, destacamos a importância de tornar a escola um ambiente 
também, com a elaboração de materiais didáticos que aproveitem o espaço escolar como espaço privilegiado para a (re)construção de saberes. 


\title{
A simulation model based on Qualitative Reasoning to Science Education
}

\begin{abstract}
The present paper presents preliminary results of research that aims to investigate didactic materials based on techniques developed in Qualitative Reasoning (QR), an area of Artificial Intelligence that uses the symbolic reasoning to represent mathematical functions without numbers and with explicitly modeled relationships of causality. Previous studies demonstrated the potential of QR to approach dynamic systems through activities that explore, with the support of simulation models, the use of hypothetical - deductive reasoning to analyze complex concepts, expanding the range of materials and activities available for teachers. Therefore, we elaborate on the modeling platform called DynaLearn (www.dynalearn.eu) a qualitative model of simulation to be used in science teaching. This software has a graphical interface, which facilitates the construction of models and the presence of the results simulation. In this paper we describe the process of construction of a qualitative model that has the objective to promote the understanding of concepts about the effect of rainfall on the behavior of a city, with respect to the consumption of drinkable water. The model represents causal relationships between climatic variables, river flow, water treatment and urban supply. In addition to having potential for use by non-deaf students in science classes, the model can also be employed in the education of the deaf because of the way it exploits visual resources.
\end{abstract}

KEYWORDS: Teaching materials. Qualitative Reasoning. Science Teaching. 


\section{AGRADECIMENTOS}

Ao Programa de Pós-Graduação em Educação em Ciências (PPGEduC) da Universidade de Brasília (UnB). Ao Instituto Federal de Educação, Ciência e Tecnologia Goiano (IF Goiano) - Campus Avançado Catalão.

\section{REFERÊNCIAS}

ALVES, F. B. Raciocínio qualitativo e desenvolvimento de raciocínio hipotéticodedutivo: uma proposta para alunos surdos. 2015. 162 f. Dissertação (Mestrado em Ensino de Ciências) - Instituto de Ciências Biológicas, Instituto de Física, Faculdade de Planaltina, Instituto de Química - Universidade de Brasília, Brasília, 2015.

BASTOS, R. C. G. Introdução à dinâmica de sistemas: proposta de disciplina a distância para curso de licenciatura em biologia, com uso de modelos qualitativos. 2014. 119 f. Dissertação (Mestrado em Ensino de Ciências) - Instituto de Ciências Biológicas, Instituto de Física, Faculdade de Planaltina, Instituto de Química, Universidade de Brasília, Brasília - DF. 2014.

BREDEWEG, B.; LIEM, J.; BEEK, W.; LINNEBANK, F.; GRACIA, J.; LOZANO, E.; WIBNER, M.; BÜHLING, R.; SALLES, P.; NOBLE, R.; ZITEK, A.; BORISOVA, P.; MIODUSER, D. DynaLearn - An Intelligent Learning Environment for Learning Conceptual Knowledge. Al Magazine, v. 34, n. 4, p. 46-65, 2013.

BREDEWEG, B.; LIEM, J.; NICOLAOU, C. Assessing learner-constructed conceptual models and simulations of dynamic systems. In: QUALITATIVE REASONING 29TH INTERNATIONAL WORKSHOP ON QUALITATIVE REASONING, 29., Amsterdam. Anais...New York, 2016.

CACHAPUZ, A.; GIL-PEREZ, D.; CARVALHO, A. M. P.; PRAIA, J.; VILCHES, A. (Orgs.). A necessária renovação do ensino das ciências. 3.a ed. São Paulo: Cortez, 2011.

CAVALCANTE, T. F. Avaliação do Uso de Modelagem Qualitativa com Apoio de Agentes Aprendizes Virtuais na Compreensão da Dinâmica de Sistemas por Alunos do Ensino Fundamental. 2015. 181f. Dissertação (Mestrado em Ensino de Ciências) - Instituto de Ciências Biológicas, Instituto de Física, Faculdade de Planaltina, Instituto de Química, Universidade de Brasília, Brasília - DF. 2015.

CROUSE, M; FORBUS, K. How Much Qualitative Reasoning is Required in Elementary School Science Test Questions?. In: QUALITATIVE REASONING 29TH INTERNATIONAL WORKSHOP ON QUALITATIVE REASONING, 29., Amsterdam. Anais...New York, 2016. 
FELTRINI, G. M. Aplicação de modelos qualitativos à educação científica de surdos. 2009. 222 f. Dissertação (Mestrado em Ensino de Ciências) - Instituto de ciências Biológicas, Instituto de Física, Instituto de Química, Universidade de Brasília, Brasília - DF. 2009.

FERREIRA, W. M.; NASCIMENTO, S. P. F.; PITANGA, A. F. Dez Anos da Lei da Libras: Um Conspecto dos Estudos Publicados nos Últimos 10 Anos nos Anais das Reuniões da Sociedade Brasileira de Química. Química Nova na Escola, v. 36, n. 3, p. 2014.

LEÃO, I. G. S. O uso de modelos de Raciocínio Qualitativo para investigar a teoria e a dinâmica de metapopulações. 2011. 135 f. Dissertação (Mestrado em Ecologia) - Instituto de Ciências Biológicas, Universidade de Brasília, Brasília - DF. 2011.

LIEM, J. Supporting Conceptual Modelling of Dynamic Systems: A Knowledge Engineering Perspective on Qualitative Reasoning. 2013. 288 f. PhD Thesis (Computer science), University of Amsterdam, 2013.

MIODUSER, D. et al. Final report on DynaLearn evaluation studies. DynaLearn, EC FP7 STREP project 231526, Deliverable D7.4, 2012.

NADOLNY, L. F. Estratégias de formação continuada para Professores de Educação Infantil: Em foco a linguagem movimento. 2010. 101 f. Dissertação (Mestrado em Educação) - Universidade Federal do Paraná, Curitiba, 2010.

PEREIRA, L. L. S.; BENITE, C. R. M.; BENITE, A. M. C. Aula de Química e Surdez: sobre Interações Pedagógicas Mediadas pela Visão. Química Nova na Escola, v. 33, n. 1, 2011.

REIS, E. S.; SILVA, L. P. O ensino das ciências naturais para alunos surdos: concepções e dificuldades dos professores da escola Aloysio Chaves Concórdia/PA. Revista do EDICC, Campinas/SP, v. 1, p.240-249, out/2012.

RESENDE, M. M. P. Avaliação do uso de modelos qualitativos como instrumento didático no ensino de ciências para estudantes surdos e ouvintes. 2010. $162 \mathrm{f}$. Dissertação (Mestrado em Ensino de Ciências) - Instituto de Ciências Biológicas, Instituto de Física, Instituto de Química, Faculdade Planaltina, Universidade de Brasília, Brasília - DF. 2010. chemical education. In: Intelligent Tutoring Systems: $7^{\text {th }}$ International 
Conference, ITS 2004, Proceedings. Lecture Notes in Computer Science Series, vol. 3220, pp. 870-872. Anais... Berlim - Heidelberg, Springer Verlag, 2004.

SOUZA, P. V. T.; GAUCHE, R.; SALLES, P. Apontamentos de um levantamento Bibliográfico, sobre inclusão, na Revista Química Nova e Química Nova na Escola entre o período de 1995 a 2016. In: Anais do 36.o Encontro de Debates sobre o Ensino de Química (36.o Edeq), 2016, Pelotas/RS. Anais...Pelotas/RS: Instituto Federal de Educação, Ciência e Tecnologia Sul-Rio-Grandense/ Universidade Federal de Pelotas, 2016.

SOUZA, P.V.T.; SALLES, P.; GAUCHE, R. Elementos para a elaboração de uma estratégia didática para o ensino de química, destinada ao aprendizado de surdos e ouvintes, baseada em Raciocínio Qualitativo. In: Anais do XVIII Encontro Nacional de Ensino de Química (XVIII Eneq), 2016, Florianópolis/SC.

Anais...Florianópolis: Instituto de Química da Universidade Federal de Santa Catarina, 2016.

SOUZA, P. V. T.; SILVA, M. R. S.; AMAURO, N. Q.; CASTRO, P. A. Ensino Médio: função propedêutica ou de formação para a vida?. In: CONGRESSO IBEROAMERICANO DE INVESTIGAÇÃO QUALITATIVA/ INTERNATIONAL SYMPOSIUM ON QUALITATIVE RESEARCH, 4., 2015, Porto. Anais... Porto, p. 184-189, 2015.

Recebido: 29 mai. 2017

Aprovado: 21 jul. 2017

DOI: $10.3895 /$ actio.v2n1.6750

Como citar:

SOUZA, P. V. T.; SALLES, P.; GAUCHE, R. Um modelo de simulação baseado em Raciocínio Qualitativo para a educação em Ciências. ACTIO, Curitiba, v. 2, n. 1, p. 162-183, jan./jul. 2017. Disponível em:

$<$ https://periodicos.utfpr.edu.br/actio>. Acesso em: XXX.

Correspondência:

Paulo Vitor Teodoro de Souza

Instituto Federal de Educação, Ciência e Tecnologia Goiano (IF Goiano) - Campus Catalão. Av. 20 de Agosto, 410, centro, Catalão, Goiás, Brasil.

Direito autoral: Este artigo está licenciado sob os termos da Licença CreativeCommons-Atribuição 4.0 Internacional. 